Wimblu, Rev. Estud. de Psicología UCR, 17(1) 2022 (Enero-Junio): 7-22 /ISSN: 1659-2107

\title{
Niveles de estrés en personal docente de los departamentos de Ciencias Sociales y Educación de la Universidad de Costa Rica, Sede de Occidente ante la situación del COVID-191
}

\author{
Stress levels in teaching staff of Social Sciences and Education \\ Departments of the Universidad de Costa Rica, Western Campus in the \\ face of COVID-19 situation
}

\author{
Livan Lizano Lizano ${ }^{2}$ \\ Roger Sánchez Alfaro ${ }^{3}$.
}

\begin{abstract}
RESUMEN
El estrés laboral es presentado como un fenómeno actual en la población docente causado por variables sociodemográficas, factores psicosociales, cultura organizacional y variables relacionadas con su labor. Este estudio tuvo como objetivo analizar los niveles de estrés laboral en el personal docente de los Departamentos de Ciencias Sociales y Educación de la Universidad de Costa Rica, Sede de Occidente en el momento de la modalidad de la virtualidad y del teletrabajo a raíz de la emergencia mundial por el COVID-19. Se realizó un estudio asociativo con una muestra de 70 docentes, a los cuales se les aplicó un cuestionario sobre datos sociodemográficos y condiciones laborales y la Escala de Estrés Percibido de Cohen. Se encontró que los niveles de estrés percibido son medios, con una media de $\mathrm{M}=25.74$ y un $84.4 \%$ de integrantes la muestra se encuentra entre los niveles medios y altos. Aunado a ello no existen diferencias significativas de estrés percibido entre los departamentos. Los resultados basados en análisis de regresión múltiple indicaron que variables como el género, la edad, si reside con adultos mayores que requieren de su cuidado y la adaptación a la modalidad virtual son predictoras en la prevalencia del estrés percibido.
\end{abstract}

Palabras clave: estrés laboral, docentes universitarios, virtualidad, teletrabajo, COVID-19.

\footnotetext{
ABSTRACT

The job stress is a current phenomenon faced by the academic population. It has been caused by many socioeconomic, and psychosocial factors, organizational culture, and some work-related elements. In fact, there was a study with the objective of analyzing the levels of stress caused in the academic population of Social Sciences and Education of the Universidad de Costa Rica, in which they took advantage from virtual modality and teleworking since COVID-19 pandemic. As a result, 70 teachers were tested by a social-active questionnaire where they answered a few questions

${ }^{1}$ Investigación elaborada en el Módulo de Psicología de los Procesos Laborales y el curso Investigación VI: Análisis Multivariado de la carrera de Psicología, Universidad de Costa Rica, Sede de Occidente, bajo la supervisión de la M.Sc. Harlen Alpízar Rojas. Correo electrónico: harlen.alpizarroja@ucr.ac.cr y Licda. Alejandra Rodríguez. Correo electrónico: alerodriguezv@gmail.com

${ }^{2}$ Universidad de Costa Rica, Sede de Occidente, San Ramón, Costa Rica. Estudiante de Psicología. Correo electrónico: livan115@outlook.com ORCID: https://orcid.org/0000-0001-6806-2222

${ }^{3}$ Universidad de Costa Rica, Sede de Occidente, San Ramón, Costa Rica. Estudiante de Psicología. Correo electrónico: resa6464@gmail.com ORCID: https://orcid.org/0000-0003-3641-5382

DOI: $10.15517 /$ WL.V17I1.49498

Recepción: 8/3/2021 Aceptación: 9/9/2021
} 
about socio-demographic reality and job conditions under Cohen's Perceived Stress Scale. For example, the levels of job stress found are regular, average $\mathrm{M}=25.74$ and the $84.4 \%$ of the people asked are between average levels and high levels.

In conclusion, the results based in analysis of multiple sectors define the variables like gender, age, whether they live with adults and older people who requires attention and the adaption to the virtual modality are predictor variables in the prevalence of stress received.

Key Words: job stress, university teachers, virtuality, teleworking, COVID-19.

\section{Introducción}

El estrés laboral aparece cuando existe la presencia de agitación mental y tensión corporal a niveles crónicos, es decir, que sobrepasan las capacidades de la persona en su contexto de trabajo, las exigencias laborales y del día a día en la labor y que pueden representar situaciones que afectan a las personas de manera negativa y producen niveles de estrés elevados. ${ }^{4}$

Barraca señala que, en los y las docentes, la interacción con estudiantes problemáticos, así como los padres de estos son algunos factores importantes que influyen en el desarrollo de estrés, el cual puede, si se presenta durante tiempos prolongados, evolucionar en el denominado Síndrome de Burnout. ${ }^{5}$ En la población en general, se suele ver a esta variable como la principal causa de estrés en profesores y profesoras de enseñanza, tanto primaria como secundaria y, debido a que las y los docentes universitarios están exentos, en gran medida, de estas problemáticas, el desarrollo de estrés es poco estudiado en este tipo de población, a pesar de que están expuestos a otros factores que se pueden considerar como estresores. Por ejemplo, en la Universidad de Costa Rica, las y los docentes universitarios suelen tener responsabilidades administrativas, de investigación, y acción social, además de procesos burocráticos y una carga laboral en proporción a su nombramiento o con el trabajo parcial en la universidad y a tiempo completo en otros espacios. También, se da que en muchas ocasiones el personal docente de la Sede de Occidente asume labores ad-honorem con el objetivo de colaborar con procesos administrativos que no se logran justificar desde sus nombramientos habituales.

A todo esto, se le puede añadir la crisis mundial a raíz de la enfermedad COVID-19 que ha forzado múltiples trabajos hacia la virtualización. A la luz de lo anterior, el presente estudio tuvo

\footnotetext{
${ }^{4}$ Magaly Cárdenas, Luz Méndez y Mónica González, «Desempeño, estrés, burnout y variables personales de los docentes universitarios», Educere 18, 60 (2014): 291-292

5 Jorge Barraca, «Emociones negativas en el profesorado universitario: burnout, estrés laboral y mobbing»,

EduPsykhé 9, 1 (2010): 88
} 
como objetivo principal analizar los niveles de estrés laboral en el personal docente de los Departamentos de Ciencias Sociales y Educación de la Universidad de Costa Rica, en su Sede de Occidente en el periodo de modalidad de la virtualidad y teletrabajo del año 2020. Asimismo, mediante esta investigación, se pretendió abordar los siguientes objetivos específicos: identificar las características demográficas que pueden funcionar como factores protectores o de riesgo que influyan en el nivel de estrés percibido por parte estos docentes, y verificar la coherencia de la Escala de Estrés percibido de Cohen en la muestra participante.

Acerca del tema del estrés laboral en docentes se han encontrado investigaciones en diversas zonas de Latinoamérica. Estos son de gran utilidad para analizar variables que pueden predecir el desarrollo de este padecimiento.

Araya y Alpízar realizaron una investigación en la Universidad de Costa Rica en la Sede de Occidente con el objetivo de estudiar la incidencia del Síndrome de Burnout en la población docente de la Sede de Occidente de la Universidad de Costa Rica y caracterizar las clases latentes según algunas variables sociodemográficas, la muestra para este estudio es de 251 profesores y se utilizó el Inventario de Burnout de Maslach (IBM), que mide el grado de las características del Síndrome de Burnout. ${ }^{6}$

Se encontró que el Síndrome de Burnout si está presente en el profesorado de la sede universitaria y que los niveles de desgaste son bajos y medios, también se concluye que el estado civil de divorcio es un factor de riesgo. Esta investigación rescata algunas variables sociodemográficas relevantes que pueden ser protectoras al estrés: el estado civil, años de experiencia en docencia, hijos e hijas, compañía en el hogar y la posibilidad de hacer ejercicio. ${ }^{7}$

Asimismo, diversos estudios apuntan a que variables sociodemográficas, factores psicosociales y la sobrecarga laboral son factores determinantes en la prevalencia del estrés laboral y, en su consecución, al Burnout. Dichos estudios fueron realizados a muestras grandes de docentes universitarios, en los cuales se destaca que los docentes con más antigüedad tienen más responsabilidades y exigencias, asimismo situaciones inesperadas en el contexto de los y las

${ }^{6}$ Carlomagno Araya y Harlen Alpízar, «Diagnóstico del Síndrome del burnout en docentes universitarios por medio del modelo de clases latentes una aplicación del Bootstrap paramétrico», Pensamiento Actual 18, 31 (2018-2019): 2434

7 Araya y Alpízar, «Diagnóstico del Síndrome del burnout en docentes universitarios por medio del modelo de clases latentes una aplicación del Bootstrap paramétrico», 24-34. 
docentes que no son capaces de manejar son factores que también favorecen a la prevalencia del estrés. 891011

Otros estudios en cambio analizaron cómo la satisfacción laboral y el aliviar las cargas laborales pueden ser factores determinantes en la poca prevalencia del estrés, en los cuales se realizó también con muestras de docentes universitarios. Se utilizaron la Escala de Estrés Percibido de Cohen (PSS) versión México, el Cuestionario de Burnout del profesorado, y la encuesta de Desempeño Magisterial. Los resultados evidencian una medida de puntuaciones relativamente baja en las escalas de estrés, asimismo los y las docentes pudieron mostrar satisfacción con sus trabajos, y sumado a esto no se muestra la prevalencia de Burnout. ${ }^{12} 13$

Por otra parte, en España se realizó un estudio donde se comprobó si variables demográficas, sociales, laborales y motivacionales estaban asociadas a los diferentes modos de afrontar el estrés laboral. El estudio es realizado con 258 docentes de la Universidad de Extremadura. Para efectos de la investigación se utilizó un Cuestionario sociodemográfico, laboral y motivacional, el cual fue diseñado con objetivo de recoger información referente a aspectos sociodemográficos, laborales y otros de carácter motivacional. También se les aplicó la Escala de Evaluación de Técnicas de Afrontamiento o C.O.P.E. que tiene como objetivo evaluar los aspectos motores o conductuales de la respuesta de estrés. ${ }^{14}$

En este estudio se encontró que un 49\% de la varianza de la variable analizada de los modos de afrontamiento se explica por factores motivacionales $(25,5 \%)$, por factores sociodemográficos $(15,5 \%)$ y por factores laborales $(8 \%)$. Con base a esto se puede concluir que estos modos de

\footnotetext{
${ }^{8}$ Patricia Vélez y Luz Mayorga, «Relación de los factores de riesgo psicosociales y el síndrome de burnout en docentes de planta de la Universidad Francisco de Paula Santander en el periodo del 2011-2012», Revista Ciencia y Cuidado 10, 2 (2013): 85-95

${ }^{9}$ Jesús Esteras, Paloma Chorot y Bonifacio Sandín, «Predicción del burnout en los docentes: Papel de los factores organizacionales, personales y sociodemográficos» Revista de Psicopatología y Psicología Clínica 19, 2 (2014): doi: https://doi.org/10.5944/rppc.vol.19.num.2.2014.13059

${ }^{10}$ Lilian González y Yudit Orta, «La Cultura Organizacional y el Síndrome de desgaste profesional en profesores universitarios» Revista Chakiñan, 1 (2017): 14-30

${ }^{11}$ María del Carmen Rodríguez-Vega, María Preciado, María Aguilar-Aldrete, Carolina Aranda-Beltrán, Silvia León-Cortés y Sergio Franco, «Causas y situaciones que inciden en el estrés laboral de profesores del CUCS-U DE G», Salud Jalisco 1, 2 (2014): 94-100

12 Magaly Cárdenas, Luz Méndez y Mónica Gonzáles, «Desempeño, estrés, Burnout y variables personales de los docentes universitarios», Educere 18, 60 (2014): 289-302

${ }^{13}$ Carlos Salazar, María Chiang, Yohana Muñoz, «Competencias docentes en la educación superior: un estudio empírico en la Universidad del Bio-Bío», Actualidades Investigativas en Educación 16, 1, (2016), $253-281$.

14 Eloísa Guerrero, «Análisis pormenorizado de los grados de burnout y técnicas de afrontamiento del estrés docente en profesorado universitario», Anales de Psicología 19, 1 (2003): 145-158
} 
afrontamiento del estrés se diferencian de acuerdo con variables como el género, el estado civil, la maternidad o paternidad, así mismo variables como las áreas de conocimiento, las categorías laborales docentes y también los inconvenientes de la profesión como docente de educación superior influyen en la afectación del estrés laboral. ${ }^{15}$

En síntesis, en la mayoría de las investigaciones se pueden encontrar niveles moderados de estrés laboral, lo que, sin embargo, no indica que los niveles de Burnout sean altos. Esto es, porque el Burnout se considera como una consecuencia de la presencia prolongada de estrés en las personas. ${ }^{16}$ Es por todo esto que la importancia del estudio radica en que aporta un estado de la cuestión sobre los niveles de estrés que los y las docentes están presentando actualmente teniendo en cuenta contexto que se vive de virtualidad y teletrabajo a raíz de la emergencia mundial por el COVID-19, así como distintas variables sociodemográficas y condiciones de trabajo del personal docente que están directamente relacionados a dichos niveles. Siguiendo esta línea, se plantea como hipótesis que las puntuaciones de la Escala de Estrés Percibido serán significativamente diferentes entre los profesores/as pertenecientes al Departamento de Educación y al de Ciencias Sociales.

Con base a los antecedentes presentados, se realizaron algunas descripciones sobre conceptos importantes que giran en torno al tema de investigación. Se puede considerar al estrés como una afectación a la homeostasis de los sistemas simpáticos, lo que lleva a reacciones fisiológicas ante cualquier estímulo nocivo, físico o psicológico. ${ }^{17}$ Para Lazarus y Folkman el estrés es la reacción que producen las amenazas que ponen en peligro el bienestar de las personas, ${ }^{18}$ estas amenazas impiden responder de manera adecuada a situaciones cotidianas. ${ }^{19}$

Bajo esta línea, Neidhart, Weinstein y Conry definen el estrés como "un elevado nivel crónico de agitación mental y tensión corporal, superior al que la capacidad de la persona puede aguantar y que le produce angustia, enfermedades o una menor capacidad para superar esas

\footnotetext{
${ }^{15}$ Eloísa Guerrero, «Análisis pormenorizado de los grados de burnout y técnicas de afrontamiento del estrés docente en profesorado universitario», 145-158.

${ }^{16}$ Carlomagno Araya y Harlen Alpízar, «Diagnóstico del Síndrome del burnout en docentes universitarios por medio del modelo de clases latentes una aplicación del Bootstrap paramétrico» Pensamiento Actual 18, 31 (2018-2019): 3233.

${ }^{17}$ Magaly Cárdenas, Luz Méndez y Mónica Gonzáles, «Desempeño, estrés, Burnout y variables personales de los docentes universitarios», Educere 18, 60 (2014): 289-302

${ }^{18}$ Richard Lazarus, Susan Folkman, Stress, Apprasial and Copying (New York: Springer Publishing Companny, Inc, 1984), 1-21.

${ }^{19}$ María Luisa Naranjo, «Una revisión teórica sobre el estrés y algunos aspectos relevantes de este en el ámbito educativo», Revista educación 33, 2 (2009): 171-190
} 
Wimblu, Rev. Estud. de Psicología UCR, 17(1) 2022 (Enero-Junio): 7-22 /ISSN: 1659-2107

situaciones". ${ }^{20}$ Naranjo menciona que existe un buen estrés, este implica todo aquello que cause placer, lo que le cause a la persona una aceptación y una armonía con sí mismo, es decir, que este estrés se manifiesta como una sensación agradable. ${ }^{21}$

Con respecto al tema del estrés, Osorio y Cárdenas ${ }^{22}$; Cárdenas et al. ${ }^{23}$ señalan que no existe una definición conceptual común, por lo cual, es válido señalar que no existe un término general para el mismo. Por esta razón, los mismos autores proponen algunas descripciones como por ejemplo que es "el resultado de demandas que exceden la capacidad de control laboral del trabajador", que es un "estado psicológico que se acompaña de síntomas físicos y disfunciones sociales; resultado de presiones físicas, mentales y sociales." o que es una "respuesta biológica y psicológica de la persona frente a demandas del medio". Estos autores hacen mención de dos exponentes importantes en este tema, que son Hans Selye, citado en Osorio y Cárdenas, quien afirma que el estrés es producto de una reacción de factores imprecisos, es decir, que puede detonarse por cualquier estímulo de amenaza. ${ }^{24} \mathrm{Y}$ al autor Robert Karasek, citado en Osorio y Cárdenas, quien plantea el estrés como un estadio a nivel interno de la persona”. ${ }^{25}$ Destacan además variables relacionados con la aparición del estrés laboral como las demandas y el control laboral, el apoyo social, problemas fisiológicos y psicológicos.

El estrés laboral puede ser descrito como un estado propio de la persona que surge por el Síndrome General de Adaptación, este se compone de tres etapas, que son la de alarma, donde el funcionamiento normal del sistema se interrumpe, la de resistencia donde se intenta volver al estado normal y el de agotamiento, donde no es posible alcanzar este estadio por la constante permanencia de alarma. Según Osorio y Cárdenas, varios autores se han llegado a referir a la causa de esta activación como tensión, no nerviosa, sino más bien relacionada las interacciones de la persona con el ambiente, incluyendo demandas y libertades de decisión, llegando a establecer el término de tensión laboral. ${ }^{26}$

\footnotetext{
${ }^{20}$ Neidhardt, Wenistein y Conry, «Seis programas para prevenir y controlar el estrés», 17.

${ }^{21}$ Naranjo, «Una revisión teórica sobre el estrés y algunos aspectos relevantes de este en el ámbito educativo», 171190.

22 Julieth Osorio, Lucila Cárdenas, «Estrés laboral: estudio de revisión», Diversitas 13, 1 (2017), 81-90.

${ }^{23}$ Magaly Cárdenas, Luz Méndez y Mónica Gonzáles, «Desempeño, estrés, Burnout y variables personales de los docentes universitarios», Educere 18, 60 (2014): 289-302

${ }^{24}$ Julieth Osorio y Lucila Cárdenas, «Estrés laboral: estudio de revisión», Diversitas 13, 1 (2017): 86.

${ }^{25}$ Osorio y Cárdenas, «Estrés laboral: estudio de revisión», 87

${ }^{26}$ Julieth Osorio y Lucila Cárdenas, «Estrés laboral: estudio de revisión», Diversitas 13, 1 (2017), 82.
} 
Osorio y Cárdenas señalan la existencia de varios modelos teóricos que explican el concepto de estrés laboral, así como la causa de este. El primer modelo es el Job-Demands control de Karasek, este propone tres cuadrantes donde se ubican cuatro tipos de trabajos determinados por el grado de control y demanda. Están los trabajos, pasivos, con bajo control y demanda; los activos, con alto control y demanda; los de baja tensión con baja demanda y mucho control y los trabajos de alta tensión, con alta demanda y poco control. El segundo modelo es el Effort Reward Imbalance de Siegrist. Este explica que el estrés se genera debido a un esfuerzo elevado, acompañado de bajas recompensas. ${ }^{27}$

En docentes el estrés puede ser causado por su ambiente laboral, es decir, por la existencia una sobrecarga laboral, exigencias en su trabajo con respecto a la enseñanza que dan día con día, presiones externas con el alumnado, padres de familia e incluso sus propios amigos y familia, sobre todo cuando no hay reconocimiento por la labor que realizan. ${ }^{28}$

Como punto cúspide del estrés laboral en docentes, se presenta el Burnout o síndrome del desgaste profesional, este es la respuesta a un estrés que es en su mayoría crónico, este se caracteriza por tener repercusiones en el estado físico y mental de la persona, además de una actitud de despersonalización en la relación con otras personas y además presenta un sentimiento de inadecuación a las tareas que se deben realizar. ${ }^{29} 30$

Otro de los temas relevantes en este estudio es la labor docente. Esta puede ser reconocida también como trabajo educativo, en el proceso de este interactúan distintos factores con la institución educativa. La labor docente se proyecta a favor de materializar los ideales educativos de la sociedad en los alumnos, esto mediante actividades sociopolíticas que tienen la finalidad de influir en la formación de distintas características en distintos aspectos en los alumnos. ${ }^{31}$ Para cumplir con esto, los profesores utilizan distintos recursos y estrategias, las cuales están en constante evolución. Un ejemplo de esto son las diversas críticas realizadas al modelo tradicional

\footnotetext{
${ }^{27}$ Osorio y Cárdenas, «Estrés laboral», 82.

${ }^{28}$ Magaly Cárdenas, Luz Méndez y Mónica Gonzáles, «Desempeño, estrés, Burnout y variables personales de los docentes universitarios» Educere 18, 60 (2014), 289-302.

29 José Antonio Ayuso, «Profesión docente y estrés laboral: una aproximación a los conceptos de Estrés Laboral y Burnout», Revista Iberoamericana De Educación 39, 3 (2006), 1-15.

${ }^{30}$ Anabella Martínez, «El síndrome de Burnout. Evolución conceptual y estado actual de la cuestión», Revista de Comunicación Vivat Academia 112 (2010), 42-80.

${ }^{31}$ Maylene Hernández, Juana María González y Tania Mayea, «La labor educativa del docente universitario desde un enfoque integral en su modo de actuación», Pedagogía y Sociedad 19, 45 (2016), 26-43.
} 
de enseñanza donde esta se limita a la teorización y generalización de información, y otro ejemplo es el creciente apoyo a la idea de promover la ideación creativa en los estudiantes, así como la enseñanza de habilidades prácticas para el futuro desempeño profesional. ${ }^{32}$

Sin embargo, el apoyo de estas ideas puede traer consigo una serie de reformulaciones en los centros educativos, reformulaciones que se convierten en posibles variables causantes de estrés para el personal docente que, sin embargo, no son las únicas. A lo largo del tiempo se han realizado diversos estudios sobre las causas de estrés en docentes y se han identificado diversos tipos de variables, entre estas, las sociodemográficas y las relacionadas con el contexto organizacional del centro educativo. En la primera categoría se pueden incluir características como el sexo, la edad, el estado civil, la paternidad, las relaciones personales, la etapa educativa donde se imparte docencia entre otros, siendo el género, de las más estudiadas y donde aún existen resultados divididos. En la segunda se pueden incluir características propias del funcionamiento de los centros educativos donde se pueden incluir el tema salarial, la interacción entre trabajadores y la carga laboral. ${ }^{33} 343536$

La sobrecarga laboral se considera relevante para el estudio debido al contexto actual de afectación pandémica. Esta se da de manera directa por un riesgo psicosocial asociado al estrés que, de mantenerse a lo largo del tiempo, propicia el desarrollo de Burnout; esta sobrecarga se da cuando en una persona recaen más responsabilidades y actividades de las que se puede hacer responsable, una sobrecarga implica una pérdida en el bienestar, en la actividad profesional y del equilibrio emocional, se relaciona también con una vivencia depresiva o desesperanzada. A su vez se relaciona con la percepción de que los esfuerzos e inversión de energía no son proporcionales con la satisfacción obtenida. La satisfacción laboral se obtiene de experiencias exitosas, como la

\footnotetext{
32 Carlos Salazar, María Chiang y Yohana Muñoz, «Competencias docentes en la educación superior: un estudio empírico en la Universidad del Bio-Bío», Actualidades Investigativas en Educación 16, 1 (2016): 283-284

33 Jorge Barraca, «Emociones negativas en el profesorado universitario: burnout, estrés laboral y mobbing», EduPsykhé 9, 1 (2010): 85-100.

${ }^{34}$ José Antonio Torres, «Análisis del grado de satisfacción del profesorado de educación secundaria en el desarrollo de su labor docente», Contextos educativos. Revista de educación, 13, (2010), 27-42.

35 Jesús Esteras, Paloma Chorot y Bonifacio Sandín, «Predicción del burnout en los docentes: Papel de los factores organizacionales, personales y sociodemográficos», Revista de Psicopatología y Psicología Clínica 19, 2 (2014): 7992.

${ }^{36}$ Carlos Salazar, María Chiang, Yohana Muñoz, «Competencias docentes en la educación superior: un estudio empírico en la Universidad del Bio-Bío», Actualidades Investigativas en Educación 16, 1, (2016), $253-281$.
} 
obtención de reconocimiento, los sentimientos de autorrealización y la sensación de pertenencia a un grupo o institución. ${ }^{37}$

Para crear esta sensación, se pueden señalar aspectos de esta misma como la subjetividad propia y la percepción, al igual que la interacción del individuo con el entorno y sus afectaciones físicas entre otros. La forma más efectiva de medir el estrés es mediante la subjetividad, esto según lo propuesto por Cohen y Karmack ${ }^{38}$, desarrolladores de la escala de estrés percibido de Cohen, quienes hablan de la existencia de escalas que utilizaban estresores objetivos como principal uso de medición, los cuales pecaron de falta de sensibilidad. El estrés percibido, por otro lado, probó tener una importante correlación con el malestar de las personas. Debido a esto, los autores señalan que hay que darle más prioridad a la forma en la que las personas interpretan sus vivencias que a las vivencias en sí.

\section{Método}

La metodología se estableció con un diseño en el cual se pudiesen comparar los Departamentos con respecto a sus niveles de estrés, además se realizaron análisis factoriales, de regresión para explicar la relevancia de variables en la predicción del estrés y validez del instrumento.

\section{Diseño metodológico}

Para esta investigación se utilizó un diseño asociativo con una estrategia comparativa donde se analizan la relación entre variables sociodemográficas y de contexto laboral, examinando las diferencias que existen entre dos grupos (docentes de los Departamentos de Ciencias Sociales y de Educación). ${ }^{39}$ Además, no se intentó modificar ninguna variable, sino comparar y analizar los dos grupos asignados. Asimismo, se tomaron dos grupos que ya están integrados, en este caso por

\footnotetext{
${ }^{37}$ Silvia Barrios, María Cecilia Arechabala y Victoria Valenzuela, «Relación entre carga laboral y burnout en enfermeras de unidades de diálisis», Enfermería Nefrológica 15, 1, (2012): 46-55.

${ }^{38}$ Sheldon Cohen, Sheldon Karmack, Robin Mermelstein, «A global measure of perceived stress», Journal of Health and Social Behavior 24, 4 (1983), 385-396.

${ }^{39}$ Manuel Alto, Juan López, Ana Benavente, «Un sistema de clasificación de los diseños de investigación en psicología», Anales de Psicología 29, 3, (2013), 1038-1059.
} 
docentes de cada departamento, por lo tanto, las unidades de análisis no se asignan al azar, ni de manera aleatoria para realizar dicha comparación. ${ }^{40}$

\section{Participantes}

La muestra del estudio fue de 70 docentes pertenecientes a los Departamentos de Ciencias Sociales y Educación de la Universidad de Costa Rica en su Sede de Occidente. Dicha muestra fue aleatoria, por lo que no se tomó en cuenta criterios de inclusión o exclusión más allá de ser docente activo en el I ciclo del año 2020 de al menos un curso en algunos de estos dos departamentos. Asimismo, se resguardaron los aspectos éticos y de confidencialidad requeridos con los sujetos para poder realizar la investigación. También se les explicó que la participación en este proyecto no presentaba ninguna clase de riesgo además de ser voluntaria, por lo que quienes participaron podían haberse retirado en cualquier momento y que sus beneficios no se fuesen a ver comprometidos en ningún caso.

\section{Instrumentos}

Se les aplicó un pequeño cuestionario sobre datos sociodemográficos y condiciones laborales para identificar variables que puedan estar asociadas al estrés laboral en medio de la virtualidad y el teletrabajo. Aunado a ello se les aplicó la Escala de Estrés Percibido de Cohen, que es de amplia utilización y que busca analizar el grado en que las situaciones de la vida son valoradas como estresantes por las personas en el último mes. Lo cual es ideal para el presente estudio por cuanto se pretende evaluar el estrés percibido por las y los docentes en la época de crisis causada por la pandemia de COVID 19 que hizo que su empleador les enviara trabajo virtual desde sus casas. El instrumento tiene versiones que cuentan con 14, 10 o 4 ítems. Para esta investigación se utilizó la versión de 14 ítems, la cual ha mostrado una consistencia interna $\alpha=.81$. 41

\footnotetext{
40 Howard White, Shagun Shabarwal, «Diseño y métodos cuasi experimentales», Síntesis metodológicas: evaluación de impacto 8 (2014): 1-16

${ }^{41}$ Shledon Cohen, Sheldon Karmack y Robin Mermelstein, «A global measure of perceived stress», Journal of Health and Social Behavior 24, 4 (1983): 385-396.
} 


\section{Procedimiento}

Antes de seleccionar el instrumento utilizado para recolectar los datos se elige el diseño a utilizar y, en efecto de la metodología de esta investigación, se opta por un diseño asociativo entre las variables que pueden estar formando parte de un modelo que comprenda cuáles de esas variables predicen mejor en su conjunto la aparición de la condición de estrés laboral, ante la situación de la virtualidad.

Luego de haber establecido el método a utilizar para la recolección de datos, se elabora el instrumento para la recolección de datos, dicho instrumento se estructuró dentro de un formulario de Google Forms. En este se incluyó el propósito de la investigación que se realizó y un consentimiento informado con los detalles de la investigación.

La elaboración de los ítems sociodemográficos fue supervisada por las profesoras asesoras de esta investigación y se tomaron en cuenta factores como la cantidad de horas del nombramiento de los docentes, si realizan trabajos ad-honorem y cuánto tiempo semanalmente le invierten a estos, el estado civil, si el/la docente tienen hijos/as, los años trabajando como docente o trabajando para la Universidad de Costa Rica, entre otras variables. En conjunto a estos datos sociodemográficos se aplicó la Escala de Estrés Percibido de Cohen. ${ }^{42}$

Las docentes asesoras de esta investigación colaboraron con la mediación de los permisos necesarios para realizar la investigación en los departamentos, así como en la supervisión del formato de aplicación de los instrumentos de recolección.

\section{Análisis}

Para el análisis de la información, los datos obtenidos se sistematizaron y se incluyeron en el software SPSS versión 23 para la comparación entre las variables. Se realizó un análisis de regresión múltiple para identificar y analizar los niveles de estrés percibido del personal docente, es decir, la variable dependiente y de qué forma las variables sociodemográficas escogidas pueden influir en esta y, hasta cierto punto, en la variación de dichos niveles de estrés.

\footnotetext{
${ }^{42}$ Cohen, Karmack y Mermelstein, «A global measure of perceived stress», 385-396.
} 


\section{Resultados}

A pesar de lo mencionado por Cohen et al. ${ }^{43}$ sobre el hecho de que la Escala de Estrés Percibido de Cohen tiene como función principal la comparación entre grupos, y no la medición, se pueden hacer ciertas inferencias en base a la estadística de normalidad, las puntuaciones brutas mínimas (0 puntos) y máximas obtenidas por la muestra (45 puntos) y la puntuación $\mathrm{Z}$ obtenida mediante el SPSS. Así pues, se puede comenzar estableciendo tres niveles de estrés percibido en base a dichas puntaciones mínimas y máximas: el primero es el nivel bajo que va desde los 0 puntos hasta los 15 y es en el que se ubica el 18.6\% ( N=13) de las y los integrantes de la muestra, el nivel medio, donde se ubican un 65,7\% (la mayoría) de las y los docentes ( $\mathrm{N}=46)$ y que va desde los 16 a los 35 , y el alto, que va desde los 36 puntos hasta los 45 y que está compuesto por un $15.7 \%$ $(\mathrm{N}=11)$ de las y los participantes, por lo que es posible señalar que alrededor de un $84.4 \%$ de quienes componen la muestra presentan niveles de estrés que van de lo medio a lo alto, a comparación del $18.6 \%$ restante, lo que resulta preocupante. Por otro lado, la puntuación media de la muestra es de 25.74 puntos, lo que significa que la mayoría de las y los docentes participantes presentan niveles medios de estrés, lo que corresponde con los datos antes brindados. Para verificar si la distribución de dichas puntuaciones es normal, se realizó la prueba K-S de normalidad, en la que el resultado reveló no significatividad $(p=0.20>0.05)$, lo que indica que la distribución en las respuestas es normal.

En cuanto a los departamentos, estos se dividieron en tres: profesores y profesoras pertenecientes a educación, ciencias sociales y quienes pertenecen a ambos departamentos. La puntuación media de cada grupo en la escala es de: 26.3, 24.9 y 28.33, respectivamente. Luego de verificar que no hay diferencia de varianzas en los tres grupos mediante la prueba de Levene $(w=$ $0.27>0.05)$ se procede a aplicar el ANOVA de un factor. Los resultados señalan que no existen diferencias significativas entre las medias de los tres grupos $(F=0.80>0.05)$. Esto lleva a concluir que el nivel de estrés percibido no se ve afectado por el departamento al que pertenecen los profesores y profesoras.

Luego de esta prueba, se realizó el Análisis de Componentes Principales como parte de una revisión de la escala a nivel estadístico, con la intención de verificar si el instrumento es unidimensional o presenta una estructura de subescalas que no han sido reportadas por los

\footnotetext{
${ }^{43}$ Ibid, 385-396.
} 


\section{निrimu}

Wimblu, Rev. Estud. de Psicología UCR, 17(1) 2022 (Enero-Junio): 7-22 /ISSN: 1659-2107

creadores del mismo. Así pues, se comenzó realizando la prueba KMO y de esfericidad de Bartlett. En la primera se obtuvo una puntuación de .88 que indica una relación entre ítems alta. En cuanto a la segunda se obtuvo un nivel de significancia de $p=.000$, lo que nos permite rechazar la hipótesis nula de que la matriz de correlaciones es en realidad una matriz de identidad, esto significa que las variables del instrumento están lo suficientemente correlacionadas como para efectuar un Análisis de Componentes Principales (ACP). En cuanto a las comunalidades, todas las variables superan la puntuación de .40, por lo cual, es posible señalar que todos los ítems de la escala presentan un buen nivel de variabilidad común.

El análisis como tal permitió extraer dos componentes. El primero, explica un 50,72\% de la varianza total, mientras que el segundo explica un $14,99 \%$, para un total de $65,71 \%$ de la varianza explicada. La cantidad de varianza no es suficiente para refutar el hecho de que la escala posea una estructura predominantemente unidimensional.

El gráfico de sedimentación permite ver de mejor manera la cantidad de varianza que aporta cada componente.

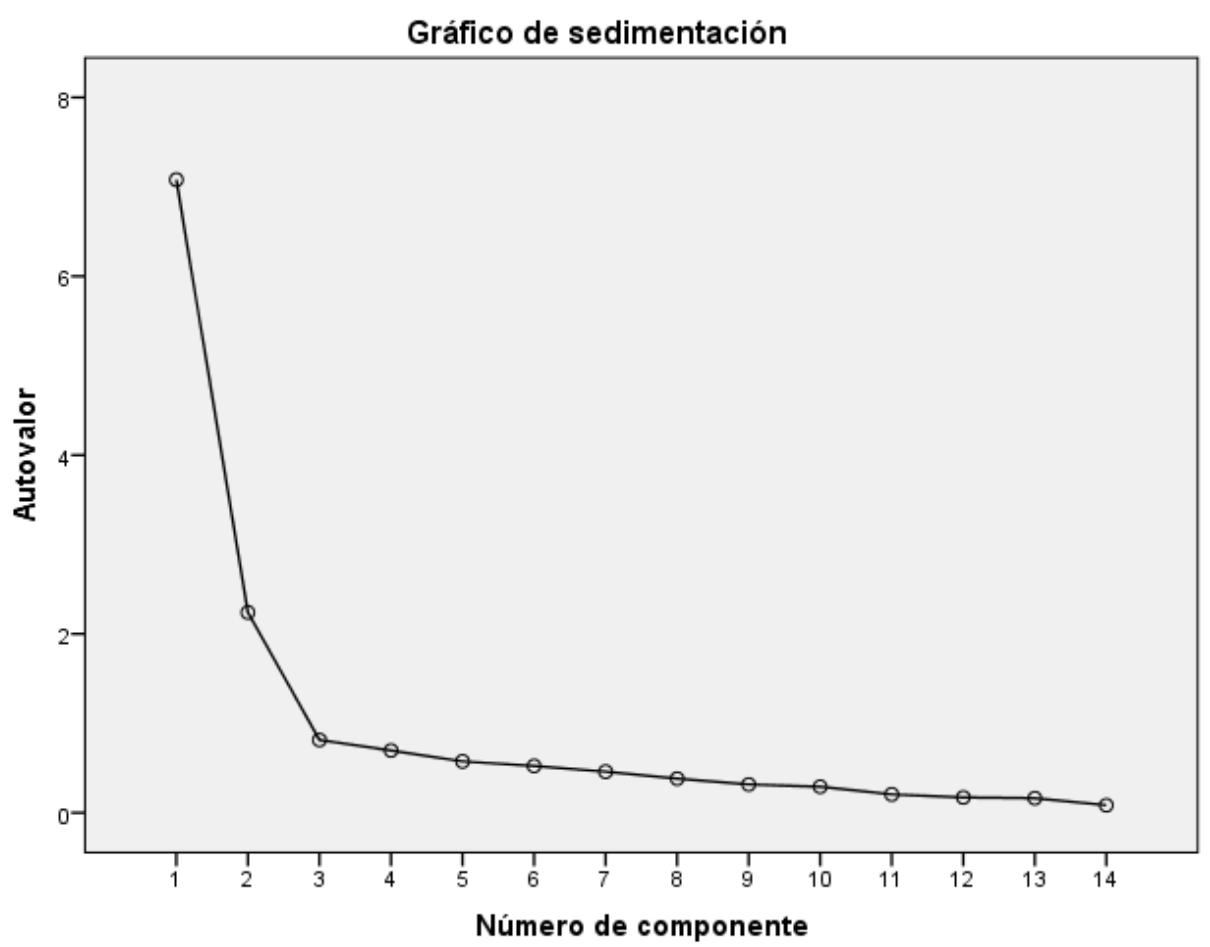

Figura 1. Gráfico de sedimentación 
Wimblu, Rev. Estud. de Psicología UCR, 17(1) 2022 (Enero-Junio): 7-22 /ISSN: 1659-2107

La tabla 1 corresponde a la matriz de patrón que indica qué variables pertenecen a cada componente y con qué magnitud lo explican. La división que es posible observar corresponde a la división realizada por los autores y corresponde a los ítems positivos, conformados por los números $4,5,6,7,9,10$ y 13 y los negativos, conformados por los números $1,2,3,8,11,12$ y 14 . Sin embargo, es posible observar que el 13 tiene carga en ambos factores, por lo que será fuente de análisis en la discusión. Debido dicha división entre factores positivos y negativos, la escala puede considerarse como unidimensional, tal como lo plantean los autores originales del instrumento. Finalmente, como parte del análisis a nivel estadístico, se considera importante señalar que la escala obtuvo un Alpha de Cronbach de $\alpha$ 0.92, que se traduce como un alto nivel de fiabilidad.

\section{Tabla 1. Matriz de patrón}

Matriz de patrón

\section{Componente}

$\underline{2}$

1. En el último mes, ¿con qué frecuencia ha estado afectado por algo que ha ocurrido inesperadamente?

2. En el último mes, ¿con qué frecuencia se ha sentido incapaz de controlar las cosas importantes en su vida?

3. En el último mes, ¿con qué frecuencia se ha sentido nervioso o estresado?

4. En el último mes, ¿con qué frecuencia ha manejado $\quad .775$ con éxito los pequeños problemas irritantes de la vida?

5.En el último mes, ¿con qué frecuencia ha sentido .853 que ha afrontado efectivamente los cambios importantes que han estado ocurriendo en su vida? 
6.En el último mes, ¿con qué frecuencia ha estado .757 seguro sobre su capacidad de manejar sus problemas personales?

7.En el último mes, ¿con qué frecuencia ha sentido .700 que las cosas van bien?

8. En el último mes, ¿con qué frecuencia ha sentido que no podía afrontar todas las cosas que tenía que hacer?

9.En el último mes, ¿con qué frecuencia ha podido .874 controlar las dificultades de su vida?

10.En el último mes, ¿con qué frecuencia se ha .635 sentido que tenía todo bajo control?

11. En el último mes, ¿con qué frecuencia ha estado enfadado/a porque las cosas que le han ocurrido estaban fuera de control?

12. En el último mes, ¿con qué frecuencia ha pensado sobre las cosas que le quedan por hacer?

13.En el último mes, ¿con qué frecuencia ha podido controlar la forma de pasar el tiempo?

14. En el último mes, ¿con qué frecuencia ha sentido que las dificultades se acumulan tanto que no puede superarlas?

Método de extracción: análisis de componentes principales. Método de rotación: Oblimin con normalización Kaiser.

a. La rotación ha convergido en 6 iteraciones. 
Para averiguar qué tipo de características sociodemográficas presentan correlación con la puntuación total de la escala se realizó un análisis de Regresión Lineal Múltiple, en el cual se introdujeron las 25 variables sociodemográficas como independientes en función a la variable dependiente: el puntaje total de la Escala de Estrés Percibido. A partir de dicho análisis se obtuvieron cuatro modelos, de los cuales se excluirán tres debido a que el nivel de explicación de la varianza total de estos no cumple con el límite recomendado (.40). El cuarto, con una $\mathrm{R}^{2}$ de .42 indica que, de las 25 variables sociodemográficas utilizadas (género, edad, departamento, carreras que se imparten, cantidad de cursos que se imparten, cantidad de horas de nombramiento, participación o no en comisiones de apoyo ad honorem, cantidad de comisiones en las que se participa, cantidad de horas ad honorem mensuales como parte de las comisiones de apoyo, participación o no en la dirección o lectura de trabajos finales de graduación, cantidad de trabajos finales dirigidos, cantidad de horas mensuales dedicadas a la dirección de dichos trabajos, cantidad de trabajos finales que deben ser leídos, cantidad de horas mensuales dedicadas a la lectura de trabajos finales, estado civil, residencia con otras personas, cantidad de personas con las que se convive, presencia de niños o adolescentes que deben ser cuidados, años de trabajo como docente en general, años de trabajo como docente de la Universidad de Costa Rica, conocimiento y uso de medios virtuales, posesión de equipo adecuado para el trabajo, residencia con adulto mayores u otras personas que requieren de cuidados y consideración sobre la propia adaptación a medios virtuales) existen cuatro que influyen de forma significativa en la puntuación total de estrés percibido, las cuales son la edad, el género, la residencia con personas adultas mayores que requieren acompañamiento y la percepción sobre la adaptación a la modalidad virtual.

Tabla 2. Resumen del Modelo

\begin{tabular}{|c|c|c|c|}
\hline & & $\underline{\mathrm{R}}$ cuadrado & Durbin- \\
\hline Modelo & $\underline{\mathrm{R} \text { cuadrado }}$ & ajustado & Watson \\
\hline 4 & .464 & .429 & 1.829 \\
\hline
\end{tabular}

El análisis de varianza de estas variables del modelo también tiene resultados significativos con una puntuación $p=.00$ (ver Tabla 3). 


\section{नriman}

Wimblu, Rev. Estud. de Psicología UCR, 17(1) 2022 (Enero-Junio): 7-22 /ISSN: 1659-2107

Tabla 3. ANOVA

\begin{tabular}{lll} 
ANOVA & & \\
\hline \hline Modelo & $\underline{\mathrm{F}}$ & $\underline{\text { Sig. }}$ \\
\hline 1 & 17.144 & .000 \\
\hline \hline
\end{tabular}

Asimismo, se demuestra que existe una relación de predicción importante entre las variables independientes del modelo 4 con la variable dependiente, en los cuales se obtuvieron puntuaciones significativas entre las variables: género del participante $(p .=0.00 ; \mathrm{VIF}=1.17)$, edad $(p .=0.00$; $\mathrm{VIF}=1.01$ ), reside con personas adultas mayores o personas de otras edades que requieren de su acompañamiento diario $(p .=0.00 ; \mathrm{VIF}=1.06) \mathrm{y}$ adaptación a la modalidad virtual $(p .=0.00 ; \mathrm{VIF}=$ 1.12). Asimismo, las cuatro variables presentan un grado de correlación moderado.

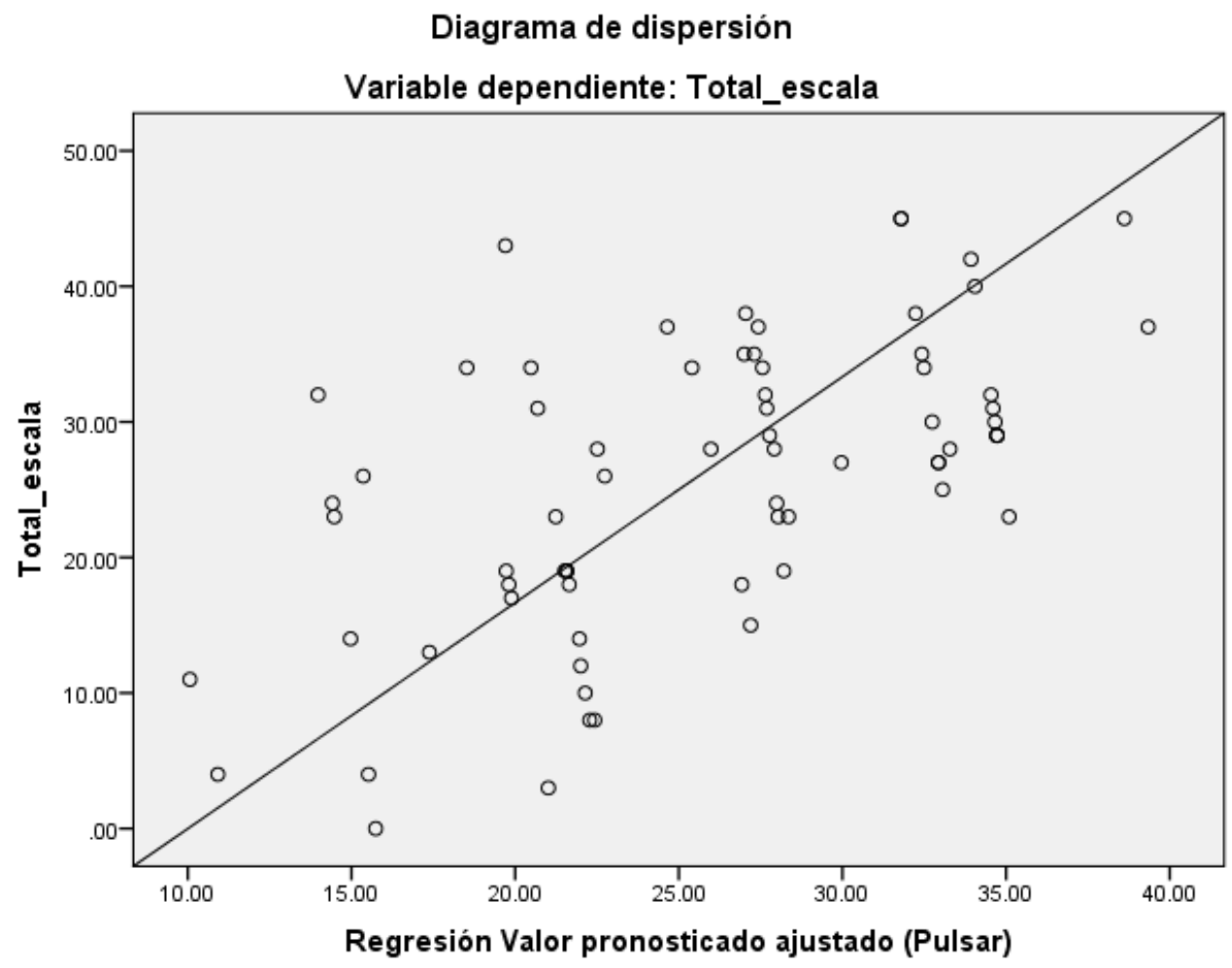

Figura 2. Diagrama de dispersión

El diagrama de dispersión muestra una relación lineal positiva parcial. Estos datos son un tanto modestos, ya que se está logrando explicar un $42 \%$ de la varianza. No obstante, pese a tener un tamaño muestra pequeño, se han logrado identificar cuatro variables importantes a considerar para la discusión. 
Por otro lado, mediante los coeficientes de regresión estandarizados, es posible conocer el grado de relevancia de las cuatro variables sociodemográficas del modelo, siendo género el que presenta mayor influencia en la varianza $(\beta=0.329)$, seguido de la edad $(\beta=-0.326)$, la residencia con personas adultas mayores que requieren de cuidados $(\beta=-0.305)$ y, por último, la adaptación a la modalidad virtual $(\beta=0.243)$. Finalmente, con respecto a la regresión lineal, la positividad o negatividad de los coeficientes también llegan a indicar que: las mujeres tienden a puntuar más alto en la escala que los hombres, los y las docentes con más edad tienden a obtener puntuaciones menores que los jóvenes, quienes no viven con personas adultas mayores que requieren de cuidados tienden a puntuar más bajo y, por último, aquellos profesores y profesoras que consideran su adaptación como regular son quienes tienen a puntuar más alto en la escala.

\section{Discusión}

A raíz de la adaptación a la virtualidad y el teletrabajo por el COVID-19, los y las docentes han tenido que comenzar a ajustarse a estos nuevos cambios, por lo que es, de cierta manera, esperable que la variable "adaptabilidad a la modalidad virtual" sea una variable predictora del estrés percibido. Asimismo, variables como el género, la edad, residir con personas adultas mayores que requieren de cuidado y la adaptabilidad a la modalidad virtual antes mencionada, también presentan diferencias significativas en puntuaciones al estrés percibido.

La muestra estuvo compuesta por 70 personas, un $64.3 \%(n=45)$ eran mujeres y un 35,7\% ( $n=25)$ eran hombres. En el análisis de regresión múltiple, la variable género reportó puntajes significativos con respecto al puntaje total de la escala $(p .=0.00)$. En un análisis de medias, esta variable reportó en el género femenino una media de $\mathrm{M}=44.08$ y en el género masculino una media de $M=32.30$. En dicho análisis de regresión se revela que el género femenino presenta una prevalencia de estrés percibido más elevada que el género masculino. Es importante destacar que en el estudio de Esteras et al. ${ }^{44}$ y el de Vélez y Mayorga ${ }^{45}$ los hombres presentaron mayores puntuaciones, pero no hubo diferencias significativas entre la variable género con respecto al estrés

\footnotetext{
${ }^{44}$ Jesús Esteras, Chorot y Sandín, «Predicción del burnout en los docentes: Papel de los factores organizacionales, personales y sociodemográficos», Revista de Psicopatología y Psicología Clínica 19, 2 (2014): 79-92.

${ }^{45}$ Patricia Vélez y Luz Mayorga, «Relación de los factores de riesgo psicosociales y el síndrome de burnout en docentes de planta de la Universidad Francisco de Paula Santander en el periodo del 2011-2012», Revista Ciencia y Cuidado 10, 2 (2013): 85-95
} 
y las variables organizacionales, personales y demográficas como predictores, pero que sí presentaban niveles de estrés alto, más no llegar a presentar burnout alto.

La población en este estudio estuvo conformada por 70 docentes, donde la media de edad fue de 41.43, en un rango de 26 a 62 años y la moda se situó en 48 años. Además, las edades se categorizaron en rangos de los 26 a los 35 años $(n=22,31,4 \%)$ con un puntaje medio en la escala de $\mathrm{M}=29.04$, de los 36 a los 55 años $(\mathrm{n}=41,58,6 \%$ ) con una media de $\mathrm{M}=25.07$ y de los 56 años en adelante $(n=7,10 \%)$ con una puntuación media de $M=19.28$. En el análisis de regresión se encontró que entre menor edad tenga la persona docente, mayor nivel de estrés presenta. En el estudio de Esteras et al, la edad no resultó ser una variable relevante para predecir las puntuaciones de estrés y burnout en el análisis de regresión múltiple ${ }^{46}$. En el estudio de Vélez y Mayorga la edad tampoco presentó ser una variable significativa para expresar los niveles altos de estrés. ${ }^{47}$ Por lo tanto, es importante observar que las personas con menor edad y las mujeres, presentan mayor puntuación en la escala de estrés percibido.

Por otra parte, en la investigación de Alpízar y Araya se encontró que hay una prevalencia de burnout en niveles bajos y medios en los docentes de la sede universitaria. Estos resultados anteceden a los resultados realizados en esta investigación, que demuestran la prevalencia de estrés percibido. Además, estos autores mencionan que una variable predictora del síndrome del burnout es el estado civil, es decir, que las personas divorciadas presentan mayor probabilidad de presentar burnout. En esta investigación, en cambio, el estado civil no representa diferencias significativas para considerarla como una variable predictora del estrés percibido. ${ }^{48}$

Es importante tomar en cuenta la situación que se está viviendo en la actualidad a raíz del COVID-19 y que, por ende, el personal docente ha tenido que irse adaptando a la virtualidad y al teletrabajo. La variable adaptación a la modalidad virtual es predictora del estrés percibido, la cual se presentó en niveles: muy bien ( $n=30,42,9 \%$ y puntuación media de $M=20.46)$, regular ( $n=40$, $57,1 \%, \mathrm{M}=27.7)$ y deficiente ( $\mathrm{n}=0,0 \%, \mathrm{M}=0)$. El $57,1 \%$ de la población indica que su adaptación

\footnotetext{
${ }^{46}$ Jesús Esteras, Chorot y Sandín, «Predicción del burnout en los docentes: Papel de los factores organizacionales, personales y sociodemográficos», Revista de Psicopatología y Psicología Clínica 19, 2 (2014): 79-92.

${ }^{47}$ Patricia Vélez y Luz Mayorga, «Relación de los factores de riesgo psicosociales y el síndrome de burnout en docentes de planta de la Universidad Francisco de Paula Santander en el periodo del 2011-2012», Revista Ciencia y Cuidado 10, 2 (2013): 85-95

${ }^{48}$ Carlomagno Araya y Harlen Alpízar, «Diagnóstico del Síndrome del burnout en docentes universitarios por medio del modelo de clases latentes una aplicación del Bootstrap paramétrico», Pensamiento Actual 18, 31 (2018-2019): 2434.
} 
ha sido de manera regular y mediante el análisis de regresión múltiple, son los que tienen mayor puntuación alta en la escala. Esto indica que gran porcentaje de la población docente ha tenido dificultades en su adaptación a la modalidad virtual y de teletrabajo, lo cual esta investigación revela que esta adaptación ha afectado a los y las docentes y que es importante tomar en cuenta esta información si se realizan investigaciones a futuro si esta condición se mantiene.

Finalmente, se recomienda tener en cuenta, para futuras investigaciones, los resultados con respecto a los niveles predominantes de estrés en la muestra. Estos pueden ser de gran ayuda para estudiar la evolución de la situación de las y los docentes con respecto a la pandemia causada por la enfermedad Covid-19 y la virtualidad, así como las consecuencias de dicha situación y que se podrían manifestar mediante el Burnout u otros padecimientos relacionados.

\section{Limitaciones}

Es importante mencionar algunas limitaciones de este estudio, como el hecho de no tener en cuenta algunas variables en el análisis y que posteriormente se consideraron importantes, como lo serían las creencias religiosas, el horario de turno, actividad laboral privada y condiciones del lugar de trabajo. Además, la situación de trabajo virtual por la pandemia de COVID-19 puede considerarse también como otra limitación importante. Sin embargo, el estudio puede servir de precedente para una futura investigación sobre burnout para estimar el impacto que el estrés constante podría tener en los docentes, donde se pueden analizar las variables que no pudieron ser incluidas.

\section{Referencias bibliográficas}

Araya, Carlomagno y Harlen Alpízar, «Diagnóstico del Síndrome del burnout en docentes universitarios por medio del modelo de clases latentes una aplicación del Bootstrap paramétrico», Pensamiento Actual 18, 31 (2018-2019): 24-34

Ato, Manuel, Juan López y Ana Benavente, «Un sistema de clasificación de los diseños de investigación en psicología», Anales de Psicología 29, 3, (2013), 1038-1059.

Ayuso, Jose Antonio, «Profesión docente y estrés laboral: una aproximación a los conceptos de Estrés Laboral y Burnout», Revista Iberoamericana De Educación 39, 3 (2006), 1-15.

Barraca, Jorge, «Emociones negativas en el profesorado universitario: burnout, estrés laboral y mobbing», EduPsykhé 9, 1 (2010): 85-100 
Wimblu, Rev. Estud. de Psicología UCR, 17(1) 2022 (Enero-Junio): 7-22 /ISSN: 1659-2107

Barrios, Silvia, Maria Cecilia Arechabala y Victoria Valenzuela, «Relación entre carga laboral y burnout en enfermeras de unidades de diálisis», Enfermería Nefrológica 15, 1, (2012), 4655.

Cárdenas, Magaly, Luz Méndez y Mónica González, «Desempeño, estrés, burnout y variables personales de los docentes universitarios», Educere 18, 60 (2014): 289-302 https://www.redalyc.org/pdf/356/35631743009.pdf

Cohen, Sheldon, Sheldon Karmack, Robin Mermelstein, «A global measure of perceived stress», Journal of Health and Social Behavior 24, 4 (1983), 385-396.

Esteras, Jesús, Paloma Chorot y Bonifacio Sandín, «Predicción del burnout en los docentes: Papel de los factores organizacionales, personales y sociodemográficos» Revista de Psicopatología y Psicología Clínica 19, 2 (2014): doi: https://doi.org/10.5944/rppc.vol.19.num.2.2014.13059

González, Lilian y Yudit Orta, «La Cultura Organizacional y el Síndrome de desgaste profesional en profesores universitarios» Revista Chakiñan, 1 (2017): 14-30. http://scielo.senescyt.gob.ec/scielo.php?script=sci_arttext\&pid=S25506722201700030001 $4 \& \operatorname{lng}=\mathrm{es} \& \mathrm{t} \operatorname{lng}=\mathrm{es}$.

Guerrero, Eloísa, «Análisis pormenorizado de los grados de burnout y técnicas de afrontamiento del estrés docente en profesorado universitario», Anales de Psicología 19, 1 (2003): 145158. https://www.um.es/analesps/v19/v19_1/14-19_1.pdf

Hernández, Maylene, Juana María González y Tania Mayea, «La labor educativa del docente universitario desde un enfoque integral en su modo de actuación», Pedagogía y Sociedad 19, 45 (2016), 26-43.

Lazarus, Richard Susan Folkman, Stress, Apprasial and Copying. New York: Springer Publishing Companny, Inc, 1984, 1-21.

Martínez, Anabella, «El síndrome de Burnout. Evolución conceptual y estado actual de la cuestión», Revista de Comunicación Vivat Academia 112 (2010), 42-80. http://www.ucm.es/info/vivataca/numeros/n112/DATOSS.htm

Naranjo, Maria Luisa, «Una revisión teórica sobre el estrés y algunos aspectos relevantes de este en el ámbito educativo», Revista educación 33, 2 (2009): 171-190. https://www.redalyc.org/pdf/440/44012058011.pdf

Neidhardt, Joseph, Malcom Weinstein y Robert Conry, Seis programas para prevenir y controlar el estrés. España: Deusto, 1989. 
Osorio, Julieth Estefanía, Lucila Cárdenas Niño, «Estrés laboral: estudio de revisión», Diversitas 13, 1 (2017), 81-90.

Rodríguez-Vega, María del Carmen, María Preciado, María Aguilar-Aldrete, Carolina ArandaBeltrán, Silvia León-Cortés y Sergio Franco, «Causas y situaciones que inciden en el estrés laboral de profesores del CUCS-U DE G», Salud Jalisco 1, 2 (2014): 94-100

Salazar, Carlos Mauricio, María Margarita Chiang, Yohana Andrea Muñoz, «Competencias docentes en la educación superior: un estudio empírico en la Universidad del Bio-Bío», Actualidades Investigativas en Educación 16, 1, (2016), 253-281.

https://dx.doi.org/10.15517/aie.v16i1.22383

Torres, Jose Antonio, «Análisis del grado de satisfacción del profesorado de educación secundaria en el desarrollo de su labor docente», Contextos educativos. Revista de educación, 13, (2010), 27-42.

Vélez, Patricia y Luz Mayorga, «Relación de los factores de riesgo psicosociales y el síndrome de burnout en docentes de planta de la Universidad Francisco de Paula Santander en el periodo del 2011-2012», Revista Ciencia y Cuidado 10, 2 (2013): 85-95.

https://dialnet.unirioja.es/servlet/articulo?codigo $=4698185$

White, Howard y Shagun Shabarwal, Diseño y métodos cuasi experimentales, (2014). 